\title{
Reduction of ER stress via a chemical chaperone prevents disease phenotypes in a mouse model of primary open angle glaucoma
}

\author{
Gulab S. Zode, ${ }^{1,2}$ Markus H. Kuehn, ${ }^{3}$ Darryl Y. Nishimura, ${ }^{1,2}$ Charles C. Searby, ${ }^{1,2}$ \\ Kabhilan Mohan, ${ }^{4}$ Sinisa D. Grozdanic, ${ }^{4}$ Kevin Bugge,, 1,2 Michael G. Anderson, ${ }^{3,5}$ \\ Abbot F. Clark, ${ }^{6}$ Edwin M. Stone, ${ }^{1,3}$ and Val C. Sheffield ${ }^{1,2,3}$
}

\begin{abstract}
${ }^{1}$ Howard Hughes Medical Institute, ${ }^{2}$ Department of Pediatrics, and ${ }^{3}$ Department of Ophthalmology and Visual Sciences, University of lowa, Iowa City, Iowa, USA. ${ }^{4}$ Department of Veterinary Clinical Sciences, College of Veterinary Medicine, lowa State University, Ames, lowa, USA. ${ }^{5}$ Molecular Physiology and Biophysics, University of lowa, lowa City, lowa, USA. ${ }^{6}$ Department of Cell Biology and Anatomy and the North Texas Eye Research Institute, University of North Texas Health Science Center at Fort Worth, Fort Worth, Texas, USA.
\end{abstract}

\begin{abstract}
Mutations in myocilin (MYOC) are the most common genetic cause of primary open angle glaucoma (POAG), but the mechanisms underlying MYOC-associated glaucoma are not fully understood. Here, we report the development of a transgenic mouse model of POAG caused by the Y437H MYOC mutation; the mice are referred to herein as $T g-M Y O C^{Y 437 H}$ mice. Analysis of adult $T g-M Y O C^{Y 437 H}$ mice, which we showed express human $M Y O C$ containing the $\mathrm{Y} 437 \mathrm{H}$ mutation within relevant eye tissues, revealed that they display glaucoma phenotypes (i.e., elevated intraocular pressure [IOP], retinal ganglion cell death, and axonal degeneration) closely resembling those seen in patients with POAG caused by the Y437H MYOC mutation. Mutant myocilin was not secreted into the aqueous humor but accumulated in the ER of the trabecular meshwork (TM), thereby inducing ER stress in the TM of Tg-MYOC ${ }^{\mathrm{Y} 437 \mathrm{H}}$ mice. Furthermore, chronic and persistent ER stress was found to be associated with TM cell death and elevation of IOP in $T g-M Y O C^{Y 437 H}$ mice. Reduction of ER stress with a chemical chaperone, phenylbutyric acid (PBA), prevented glaucoma phenotypes in $T g$ - $M Y O C^{Y 437 H}$ mice by promoting the secretion of mutant myocilin in the aqueous humor and by decreasing intracellular accumulation of myocilin in the ER, thus preventing TM cell death. These results demonstrate that ER stress is linked to the pathogenesis of POAG and may be a target for treatment in human patients.
\end{abstract}

\section{Introduction}

Glaucoma is a chronic optic neuropathy, characterized by progressive loss of retinal ganglion cell (RGC) axons, with the resultant irreversible loss of vision (1). Primary open angle glaucoma (POAG) is the most common form of glaucoma. A major risk factor for glaucoma is elevated intraocular pressure (IOP). Elevated IOP is caused by increased resistance to aqueous humor outflow through the structures of trabecular meshwork (TM). Aqueous humor is made by ciliary body, circulates the anterior chamber, and drains through the network of TM. In most glaucoma cases, there is increased resistance to aqueous humor at the TM. The molecular pathways that lead to increased resistance in TM are not well understood.

Mutations in the myocilin (MYOC) gene, which encodes a protein expressed abundantly in the TM, are the most common genetically defined cause of glaucoma, responsible for approximately $4 \%$ of POAG and most cases of autosomal dominant juvenile-onset open-angle glaucoma (2). Myocilin is expressed in many ocular tissues, including TM cells and the ciliary body, that are capable of secreting myocilin into the aqueous humor (3). Approximately 70 different myocilin mutations have been identified, and the majority of these mutations are within coding regions of the olfactomedin domain, but the significance of these mutations is unknown. The age of onset of glaucoma differs among these mutations. The Y437H MYOC mutation is

Conflict of interest: The authors have declared that no conflict of interest exists. Citation for this article: J Clin Invest. 2011;121(9):3542-3553. doi:10.1172/JCI58183. associated with elevated IOP and development of glaucoma in the second decade of life (4). The in vivo mechanisms by which mutant MYOC elevates IOP are not well understood.

Attempts to generate a MYOC mouse model that closely mimics human glaucoma have not been successful. Hemizygous (5) or homozygous (6) deletion of the MYOC gene is not associated with glaucoma. Moreover, overexpression (7) or knockout (8) of Myoc in mice does not produce glaucoma. These findings indicate that MYOC is not required for the physiological regulation of IOP and support the hypothesis that glaucoma is the result of a gain-offunction or novel property of mutant MYOC. When previously tested in a knockin mouse model, mutant mouse Myoc did not result in glaucomatous ocular changes (9). A recent study indicates that expression of only mutant human and not mouse Myoc may produce glaucoma in mice (10). This difference seems to be due to a peroxisomal targeting signal present at the carboxyl terminus of human MYOC that is absent in mouse Myoc. The presence of this signal targets mutant MYOC to peroxisomes where it may accumulate and contribute to cellular stress. Cell culture studies indicate that mutant MYOC is misfolded and accumulates intracellularly as soluble and insoluble aggregates, accompanied by ER stress (11-13). Cells can activate a cytoprotective signal response known as the unfolded protein response (UPR) in response to ER stress (14). UPR activation corrects misfolding, prevents translation of misfolded proteins, and enhances degradation of misfolded proteins. Excessive and sustained ER stress can trigger apoptotic cell death in neurological disorders and may be responsible for TM cellular dysfunction contributing to elevated IOP. 

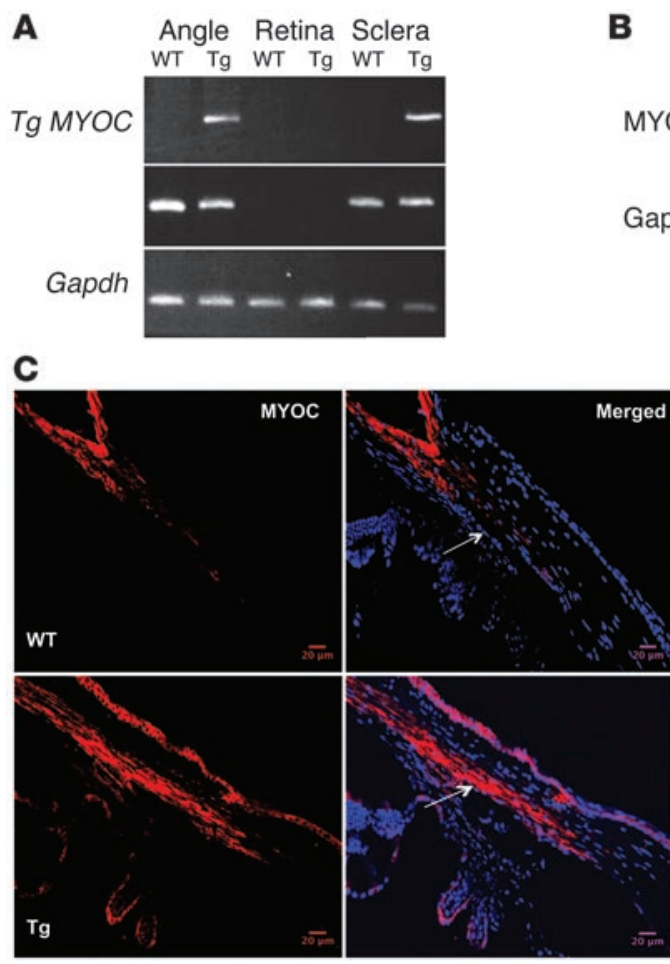

B Angle Retina Sclera

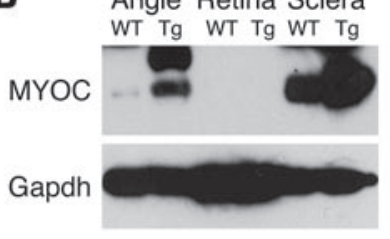

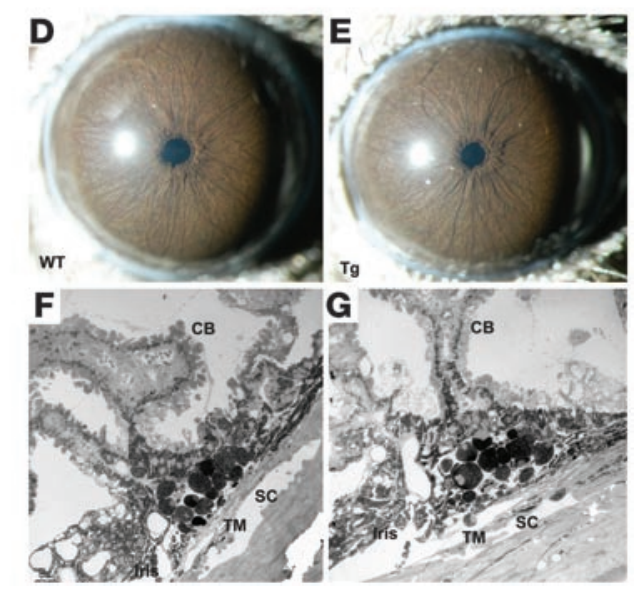

\section{Figure 1}

Increased transgene expression in TM of Tg-MYOC ${ }^{Y 437 H}$ mice. Transgene (Y437H MYOC) and WT MYOC expression were examined in 6-monthold WT and Tg-MYOC ${ }^{Y 437 H}$ littermates by (A) RT-PCR, (B) Western blot analysis, and (C) immunostaining. PCR products were sequenced to confirm the presence of the transgene. Transgene and WT MYOC were expressed only in the iridocorneal angle and sclera of $T g-M Y O C^{Y 437 H}$ mice but were absent in the retina of WT and $T g-M_{Y O C}{ }^{437 H}$ mice $(n=3)$. MYOC protein levels were increased in angle and sclera of $T g-M Y O C^{Y 437 H}$ mice compared with WT littermates. Note that MYOC protein was absent in retinal lysates in $T g-M Y O C^{\gamma 437 H}$ and WT mice ( $n=3$ ). GAPDH was used as a loading control. Immunostaining revealed that MYOC was localized to TM and is increased in $T g-M Y O C^{1437 H}$ mice (bottom panels) compared with WT littermates (top panels). The TM is shown by the arrow. Scale bars: $20 \mu \mathrm{m}$. Open iridocorneal angle and normal morphology of anterior chamber structures in $\mathrm{Tg}-\mathrm{MYOC}^{\mathrm{Y} 437 \mathrm{H}}$ mice (D-G). Slit lamp examination of anterior chambers of 6-month-old (D) WT mice and (E) Tg$M_{Y O C}{ }^{Y 437 H}$ mice reveal no abnormalities in the iris, cornea, and lens. TEM images of the iridocorneal angle of 12-month-old (F) $T g$ - $M Y O C^{Y 437 H}$ mice were compared with $(\mathbf{G})$ WT littermates. The iridocorneal angle is open in $T g$-MYOC $C^{Y 437 H}$ mice $(n=6)$ compared with WT littermates $(n=5)$. Iris, ciliary body (CB), TM, and Schlemm canal (SC) are shown.

The objectives of this study were to develop a murine transgenic model of mutant human MYOC and to utilize this model to investigate the underlying pathogenic mechanisms of glaucoma.

\section{Results}

Mutant myocilin is expressed in the TM of Tg-MYOC ${ }^{Y 437 H}$ mice. We generated transgenic mice carrying a mutation of human MYOC $(Y 437 H)$ under the control of the CMV promoter (Figure 1 and Supplemental Figure 1; supplemental material available online with this article; doi:10.1172/JCI58183DS1) and examined the glaucoma phenotypes in $86 \mathrm{Tg}-\mathrm{MYOC}^{\mathrm{Y} 437 \mathrm{H}}$ and $62 \mathrm{WT}$ littermates (Figure 2). The Y437H mutation was selected, as it is associated with relatively early onset of glaucoma in humans. The transgene is expressed and MYOC protein is observed in the iridocorneal angle and sclera of Tg-MYOC $\mathrm{C}^{\mathrm{Y} 337 \mathrm{H}}$ mice (Figure 1, A and B). Of note, mutant MYOC mRNA and protein are absent in the retina, which is similar to the expression pattern of endogenous Myoc. Immunostaining for MYOC revealed that MYOC is increased and accumulates in the TM of $T g-M Y O C^{Y 437 H}$ mice compared with WT littermates (Figure 1C).

Examination of the anterior segments of eyes from control and $\mathrm{Tg}$-MYOC ${ }^{Y 437 H}$ mice revealed that mutant and WT eyes were indistinguishable at all ages (Figure 1, D-G). Transmission electron microscopy (TEM) analysis revealed that the iridocorneal angle of $\mathrm{Tg}-\mathrm{MYOC}^{\mathrm{Y} 437 \mathrm{H}}$ mice is open and the mice do not have abnormalities of the iris, ciliary body, or cornea (Figure 1, D-G). Furthermore, gonioscopy examination of the iridocorneal angle of $\mathrm{Tg}-\mathrm{MYOC} \mathrm{C}^{\mathrm{Y} 337 \mathrm{H}}$ mice revealed that the angle is open and free of debris or other abnormalities (Supplemental Figure 2).

$T g$-MYOC ${ }^{Y 437 H}$ mice exhibit glaucoma phenotypes. In humans, the Y437H MYOC mutation is associated with elevated IOP and development of glaucoma in the second decade of life (4). We determined whether overexpression of mutant MYOC elevates IOP in $\mathrm{Tg}-\mathrm{MYOC}^{\mathrm{Y} 437 \mathrm{H}}$ mice (Figure $2 \mathrm{~A}$ ). Starting at 3 months of age, $\mathrm{Tg}$ $M_{Y O C}{ }^{Y 437 H}$ mice demonstrate significant elevation of IOP compared with WT littermates. It has been hypothesized that diurnal variation in IOP is higher in glaucoma patients (15). Tg-MYOC $\mathrm{C}^{\mathrm{Y} 37 \mathrm{H}}$ mice also show diurnal rhythms, with IOP being the highest during the night. Starting at 3 months of age, differences in IOP between $T g-M Y O C^{Y 437 H}$ and WT littermates were significantly higher at night $\left(14.1 \mathrm{mmHg}\right.$ in WT vs. $20.3 \mathrm{mmHg}$ in $\mathrm{Tg}-\mathrm{MYOC}^{Y 437 H}$; $P<0.001)$ compared with daytime $(12 \mathrm{mmHg}$ in WT vs. $15 \mathrm{mmHg}$ in $\left.\mathrm{Tg}-\mathrm{MYOC}^{\mathrm{Y4} 37 \mathrm{H}} ; \mathrm{P}<0.001\right)$. Neither daytime nor nocturnal IOP were affected in 1-month-old $\mathrm{Tg}$-MYOC ${ }^{\mathrm{Y} 437 \mathrm{H}}$ mice. $\mathrm{Tg}$ - $M Y O \mathrm{C}^{\mathrm{Y} 437 \mathrm{H}}$ mice develop elevated IOP between 2 and 3 months, which corre- 

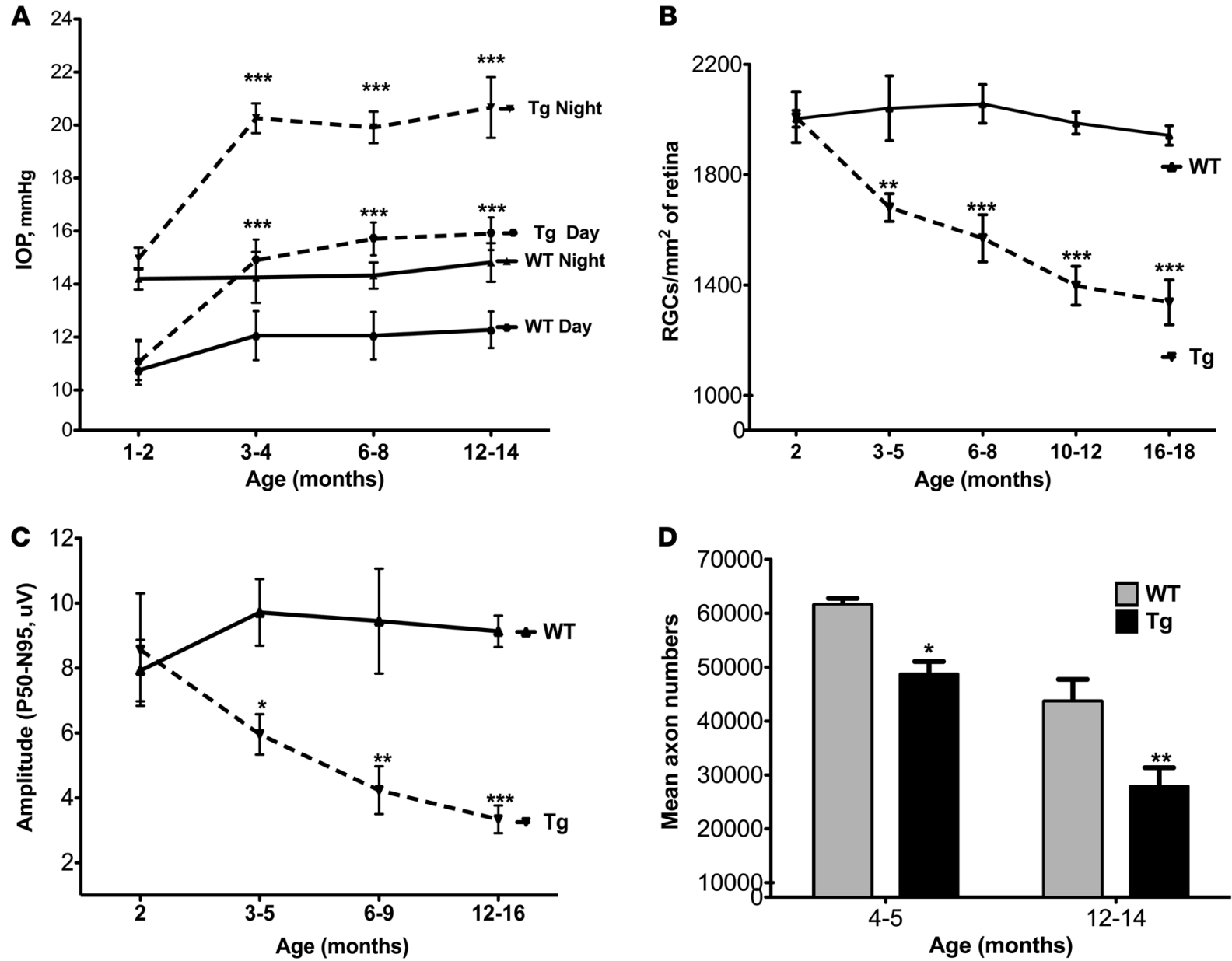

Figure 2

Glaucoma phenotypes of $T g-M Y O C^{4437 H}$ mice. (A) Elevated IOP in Tg-MYOC ${ }^{437 H}$ mice. IOP of Tg-MYOC ${ }^{\text {437H }}(n=69)$ and WT littermates $(n=62)$ were measured day and night at various ages. (B) Progressive RGC loss in $T g-M Y O C^{\gamma 437 H}$ mice. Mean RGC numbers were counted from whole-mount $\gamma$-synuclein staining of retina in $T g-M Y O C^{Y 437 H}(n=40)$ and WT littermates $(n=30)$. (C) Functional deficit in RGCs of Tg-MYOC ${ }^{\gamma 437 H}$ mice. PERG amplitudes (P50-N95) in Tg-MYOC ${ }^{437 H}(n=20)$ were compared with WT littermates $(n=14)$ at various ages. (D) Progressive optic nerve degeneration in $\mathrm{Tg}-\mathrm{MYOC}^{\mathrm{Y} 437 \mathrm{H}}$ mice. Optic nerve sections were stained with PPD, and mean axon counts in $T g-M Y O C^{Y 437 H}(n=15)$ were compared with those of WT littermates $(n=12)$. Data are shown as mean \pm SEM. ${ }^{*} P<0.05 ;{ }^{* \star} P<0.005 ;{ }^{* \star *} P<0.0001$ versus WT.

lates temporally with increased MYOC levels in these mice (data not shown). It is known that corneal thickness influences measurement of IOP (16); therefore, we measured central corneal thickness. The central corneal thickness in $T g-M Y O C^{Y 437 H}$ mice was similar to that of WT littermates (Supplemental Figure 3), indicating that overexpression of mutant MYOC does not affect corneal thickness.

We next determined whether elevation of IOP in $T g-M Y O C^{Y 437 H}$ mice would lead to RGC loss by performing whole-mount $\gamma$-synuclein staining of retinas (Supplemental Figure 4A). $\mathrm{Tg}-\mathrm{MYOC}^{\mathrm{Y} 437 \mathrm{H}}$ mice lost $17.6 \%$ of RGCs by $3-5$ months of age and $30 \%$ of RGCs by $12-14$ months of age compared with WT littermates (Figure $2 \mathrm{~B})$. We investigated functional deficits in RGCs using pattern electroretinography (PERG), a technique that selectively measures RGC function. $\mathrm{Tg}-\mathrm{MYOC} \mathrm{C}^{\mathrm{Y} 437 \mathrm{H}}$ mice demonstrate $38.7 \%$ reduction in PERG amplitude at 3-5 months and 63.7\% reduction at $12-16$ months compared with WT littermates (Figure 2C). Both RGCs and PERG amplitudes were normal in 2-month-old Tg-MYOC ${ }^{Y 437 H}$ mice compared with WT littermates. Ganzfeld ERGs (data not shown) and histological sections of retina of $\mathrm{Tg}-M Y O C^{Y 437 H}$ mice were similar to those of WT littermates (Supplemental Figure 4B), indicating that other layers of the retina of $\mathrm{Tg}-\mathrm{MYOC}^{\mathrm{Y} 437 \mathrm{H}}$ are not affected. These findings indicate specific and progressive structural and functional loss of RGCs in $\mathrm{Tg}-M Y O \mathrm{C}^{\mathrm{Y} 437 \mathrm{H}}$ mice.

We evaluated optic nerve axonal degeneration by staining with p-phenylenediamine (Supplemental Figure 5) and quantitated optic nerve axons. Tg-MYOC ${ }^{Y 437 H}$ mice lose $20.8 \%$ of axons by $4-5$ months of age and $36.1 \%$ by $12-14$ months of age compared with WT littermates, indicating progressive neuronal degeneration (Figure 2D). Together, these findings demonstrate that $T g-M Y O C^{Y 437 H}$ mice exhibit glaucoma phenotypes that closely resemble those of POAG patients with heterozygous MYOC mutations.

Mutant MYOC accumulates in the ER and induces ER stress in the TM of $\mathrm{Tg}-\mathrm{MYOC}^{\mathrm{Y} 37 \mathrm{H}}$ mice. Previous in vitro studies indicated that mutant MYOC is misfolded and is not secreted, leading to induction of ER stress $(11-13,17)$. To alleviate such cellular stress, eukaryotic cells activate a cytoprotective response known as the ER stress response or the UPR. Activation of the UPR involves sensing of ER stress via PERK, ATF- $6 \alpha$, and IRE1. To resolve the ER stress, these ER 

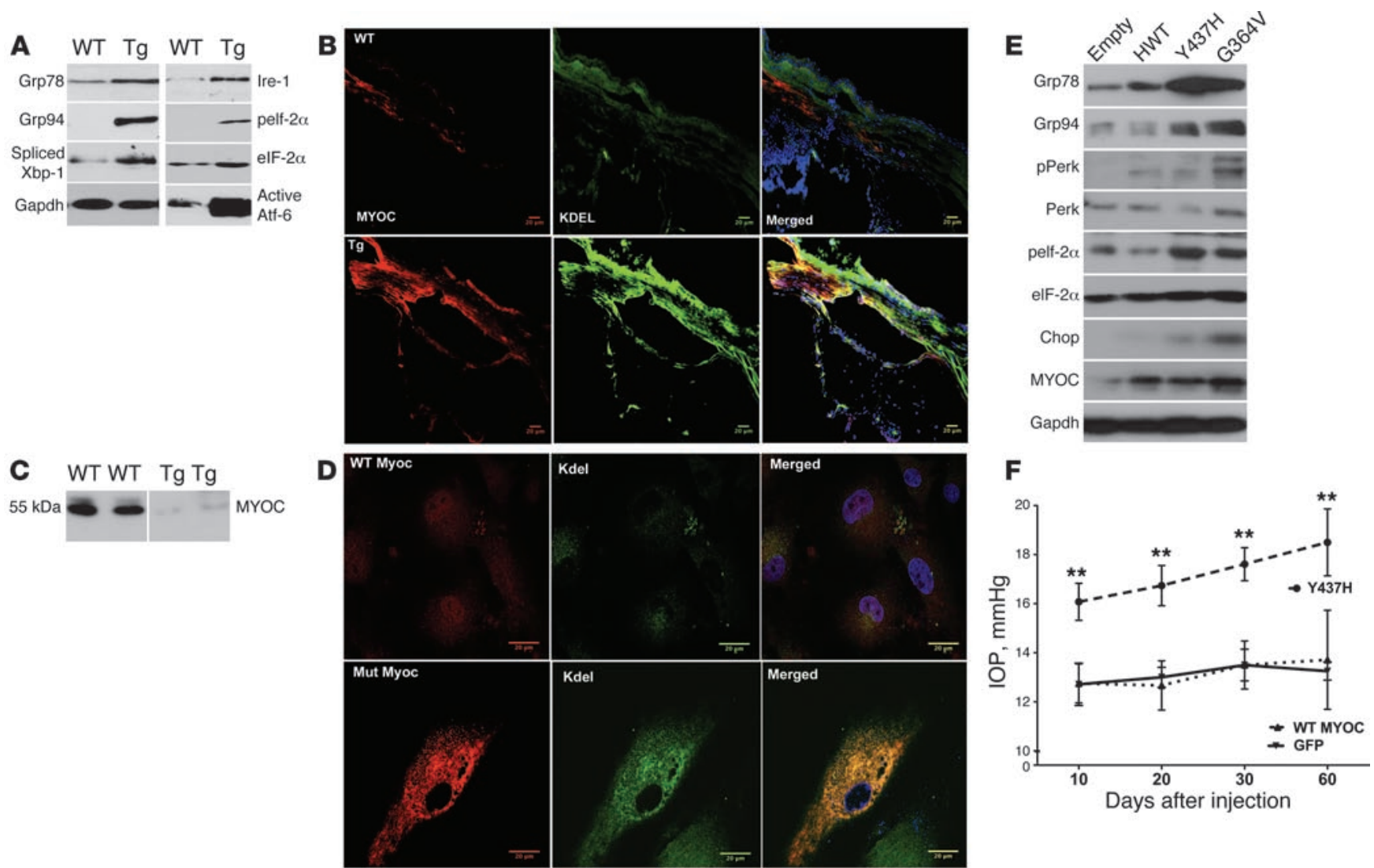

Figure 3

Mutant MYOC accumulates in the ER, induces ER stress, and activates UPR. (A) Increased ER stress markers including Ire1, Grp78, Grp94, spliced Xbp-1, activated Atf- $6 \alpha$, and pelF-2 $\alpha$ were detected in 6-month-old Tg-MYOC ${ }^{Y 437 H}$ anterior segment tissues by Western blot. These markers were also increased in 12-month-old $\mathrm{Tg}-\mathrm{MYOC}^{\mathrm{Y} 437 \mathrm{H}}$ mice. (B) Immunostaining for KDEL (recognizes Grp78 and Grp94) in the iridocorneal sections of WT and $\mathrm{Tg}-\mathrm{MYOC}^{\mathrm{Y} 437 \mathrm{H}}$ mice demonstrated increased ER stress marker localization in the TM of 6-month-old $\mathrm{Tg}$ - $M Y O \mathrm{C}^{\mathrm{Y} 437 \mathrm{H}}$ mice compared with WT littermates $(n=4)$. (C) Decreased myocilin secretion in the aqueous humor of $T g-M Y O C^{Y 437 H}$ mice. Representative Western blot of myocilin secretion in aqueous humor of $T g-M Y O C^{4437 H}$ mice is compared with that in WT mice. ( $n=6$ ). (D) Mutant MYOC accumulates in the ER of primary TM cells. Colocalization of MYOC (red) with KDEL (green) was examined using immunostaining and confocal imaging in TM cells $(n=4)$. Scale bars: $20 \mu \mathrm{m}$. (E and F) Expression of mutant MYOC in the TM induces ER stress and elevates IOP. WT MYOC, Y437H MYOC, and G364V MYOC were expressed in the TM by adenoviral injections. Western blots of ER stress markers were examined in the iridocorneal angle tissues after 24 hours of injections (E), and IOP was examined (F). ${ }^{* \star} P<0.005$ versus WT, $n=12$ WT MYOC, and 20 Y437HMYOC. Data are shown as mean \pm SEM.

stress sensors activate downstream signaling including induction of ER chaperones (GRP78, GRP94), alternative splicing of XBP-1, and phosphorylation of elF-2 $\alpha(14,18)$. We examined whether mutant $M Y O C$ induces ER stress and UPR activation in $T g-M Y O C^{Y 437 H}$ mice (Figure 3, A and B). Tg-MYOC ${ }^{Y 437 H}$ mice show increased Grp78, Grp94, phosphorylated elF- $2 \alpha$, active Atf- $6 \alpha$, Ire1, and spliced $\mathrm{Xbp}-1$ levels in eye anterior segment lysates (Figure 3A). Increased immunostaining for ER chaperones, Grp78, and Grp94 (recognized by KDEL antibody) in the TM of $T g-M Y O C^{Y 437 H}$ mice indicates induction of ER stress (Figure $3 \mathrm{~B}$ ). Together, these findings show that UPR is activated in the TM of Tg-MYOC ${ }^{Y 437 H}$ mice.

We investigated whether mutant MYOC induces ER stress and activates UPR in human TM cells by expressing WT, Y437H MYOC, and G364V MYOC. Expression of only mutant (Y437H and G364V) and not WT MYOC increases ER stress markers Grp78, Grp94, and phosphorylated elF-2 $\alpha$ (Supplemental Figure $6, A$ and B). Previous cell culture studies indicate that mutations in myocilin inhibit its secretion and result in intracellular soluble and insoluble aggregate formation $(11,12,17,19)$. Analysis of aqueous humor demonstrated little or no detectable myocilin in the aqueous humor of $\mathrm{Tg}$-MYOC ${ }^{\mathrm{Y} 437 H}$ mice compared with WT littermates, indicating inhibition of myocilin secretion due to expression of mutant myocilin (Figure 3C). Colocalization studies of MYOC with ER marker antibody KDEL or GRP78 demonstrated that WT MYOC associates transiently with the ER (Figure 3D and Supplemental Figure 7). However, mutant MYOC accumulates in the ER, as indicated by its complete colocalization with KDEL or GRP78 in vitro (Figure 3D) and in vivo (Figure 3B). Similarly, ER stress markers (KDEL) are colocalized to myocilin in the TM of Tg-MYO$\mathrm{C}^{Y 437 H}$ mice compared with WT littermates, indicating accumulation of myocilin in the ER of TM cells.

In order to determine whether overexpression of WT MYOC or only mutant MYOC induces ER stress and elevates IOP in vivo, we expressed WT or Y437HMYOC in the TM by adenoviral injections into the anterior chamber of normal C57BL/6J mice (Figure 3, E and F). Y437H MYOC significantly elevates IOP at 10, 20, 30, and 
A
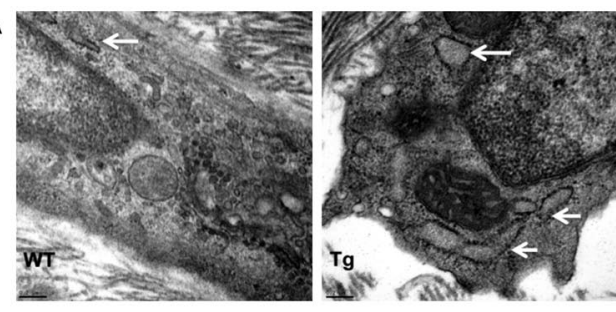

B

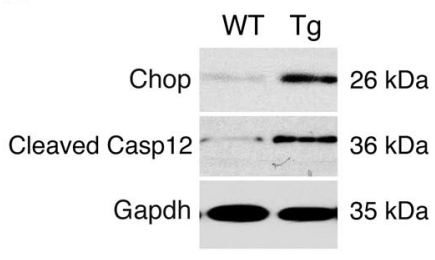

C

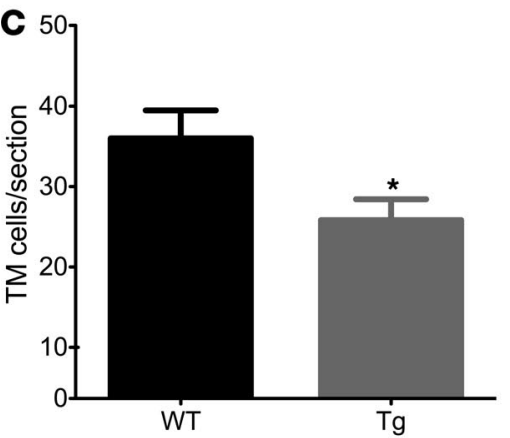

D

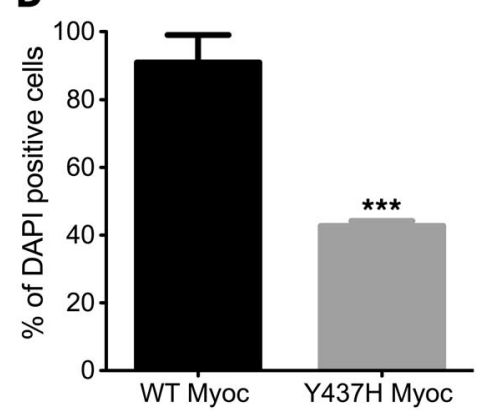

Figure 4

Mutant MYOC upregulates ER stress-induced apoptotic proteins, which is associated with loss of TM. (A) Distention of rER in the TM of $\mathrm{Tg}-M Y O C^{Y 437 H}$ mice. TEM of both genotypes demonstrated that rER (arrows) of 12-month-old $\mathrm{Tg}-M Y O \mathrm{C}^{\mathrm{Y} 437 \mathrm{H}}$ was dilated compared with that of WT littermates. Similar findings were observed in 6-month-old $\mathrm{Tg}-\mathrm{MYOC}^{\mathrm{Y} 437 \mathrm{H}}$ mice. Scale bars: $0.2 \mu \mathrm{m}$. (B) Induction of ER stress-initiated apoptotic proteins in 12-month-old $\mathrm{Tg}-\mathrm{MYOC}^{\mathrm{Y} 437 \mathrm{H}}$ mice. Western blot analysis of anterior segment tissue lysate for ER stress-induced apoptotic proteins Chop and cleaved caspase $12(n=3)$. (C) Loss of TM cells in 12-month-old $T g-M_{Y O C}{ }^{Y 437 H}$ mice examined by TEM analysis. Data are shown as an average number of TM cells/iridocorneal angle sections of WT $(n=5)$ and Tg-MYOC ${ }^{\text {437H }}$ mice $(n=6)$. Data are shown as mean \pm SEM. ${ }^{*} P<0.02$ versus WT. (D) Y437H MYOC leads to TM cell death in cultured TM cells. Percentage of DAPI-positive cells expressing either WT or $\mathrm{Y} 437 \mathrm{H}$ MYOC represented over control cells expressing empty virus. Data are shown as mean \pm SEM. $n=4$. ${ }^{\star \star *} P<0.0004$.

60 days after injection compared with GFP or WT MYOC (Figure 3F). Mice expressing Y437H MYOC also show significant RGC loss 60 days after injection (Supplemental Figure 8). Mice injected with adenovirus harboring either Y437H or G364V mutants of human myocilin did not have elevated IOP after 24 hours of injections (data not shown). However, Western blot analysis of iridocorneal angle tissues from these mice demonstrated that Y437H or G364V mutants of human myocilin induce ER stress, as evident from increased phosphorylation of Perk and elF- $2 \alpha$ as well as increased levels of Grp78, Grp94, and Chop compared with WT or empty adenovirus-injected mice (Figure 3E). These ER stress markers were also increased in the iridocorneal angle tissues of mice after 1 month of injection with mutant myocilin compared with WT myocilin-injected mice (Supplemental Figure 9) These data indicate that expression of mutant myocilin (Y437H or G364V) but not WT myocilin induces ER stress. In addition, these data also suggest that ER stress is induced by mutant myocilin prior to IOP elevation.

The ER stress-initiated apoptotic pathway is activated in Tg$M Y O C^{Y 437 H}$ mice, which may lead to elevated IOP. If the UPR adaptive response is not sufficient to resolve defects in protein folding, ER dysfunction can lead to apoptotic cell death (18). We determined whether induction of ER stress would lead to TM abnormalities and cell death in Tg-MYOC ${ }^{Y 437 H}$ mice by TEM morphological examination. $T g-M Y O C^{Y 437 H}$ mice show rough
ER (rER) distention in TM cells compared with WT (Figure 4A). Excessive and sustained UPR activation triggers apoptotic cell death via induction of CHOP and activation of ERspecific caspase 12 in neurological disorders (14). 12-month-old Tg-MYOC ${ }^{Y 437 H}$ mice also showed induction of Chop and activation of caspase 12 in the TM (Figure 4B). In addition, TEM analysis demonstrated significant loss of TM cells in 12-month-old Tg-MYOC ${ }^{Y 437 H}$ mice (Figure 4C, Figure 1, E and F, and Supplemental Figure 10). Quantitation of DAPIpositive cells indicated loss of cultured TM cells expressing mutant MYOC compared with cells expressing either empty virus or WT MYOC (Figure 4D). Together, these findings indicate that ER stress-induced apoptotic pathway is associated with TM loss, thus leading to elevated IOP.

Chemical induction of ER stress in the TM elevates $I O P$. We also sought to determine whether induction of ER stress alone is sufficient to elevate IOP. Tunicamycin, an antibiotic that inhibits $\mathrm{N}$-glycosylation proteins in the ER, has been widely used to induce ER stress in both in vivo and in vitro studies (14). We chemically induced ER stress in normal C57BL/6J mice by anterior chamber injections of tunicamycin $(0.03,0.3$, and $3 \mu \mathrm{g} /$ eye $)$ and measured IOP 3,10 , and 20 days after injection. Tunicamycin injection elevates IOP significantly in a dose-dependent manner (Figure 5A). We next examined ER stress markers in the iridocorneal angle tissues by Western blot analysis 24 hours after injections (Figure 5B). Mice injected with tunicamycin induced ER stress markers, including levels of Grp78, Grp94, Chop, and phosphorylated elF-2 $\alpha$, but did not elevate IOP 24 hours after injections (Supplemental Figure 11). These findings indicate that chemical induction of ER stress elevates IOP in vivo.

The chemical chaperone, $P B A$, rescues glaucoma phenotypes of $T g$ $M_{Y O C^{Y 437 H}}$ mice. We hypothesized that relieving ER stress in Tg$M Y O C^{Y 437 H}$ mice would rescue glaucoma. Phenylbutyric acid (PBA), a small chemical chaperone, has been shown to reduce ER stress in both in vivo and in vitro studies (20). We treated WT and $T g$ $M Y O C^{Y 437 H}$ mice (2-month-old) by adding PBA to the drinking water $(20 \mathrm{mM}, \sim 10 \mathrm{mg} / \mathrm{mouse} / \mathrm{d})$ for 5 weeks and compared glaucoma phenotypes with those of untreated littermates. Treatment of WT mice with PBA did not affect their IOP (Figure 6A). However, PBA treatment prevented the development of elevated nocturnal IOP in Tg-MYOC ${ }^{Y 437 H}$ mice $(14.1 \mathrm{mmHg}$ in treated; $n=18$ vs. $20.28 \mathrm{mmHg}$ in untreated; $n=14$ ) and maintained IOP at levels comparable to those of WT littermates (Figure 6A). PBA was also effective in significantly reducing elevated IOP in older $\mathrm{Tg}$ $M Y O C^{Y 437 H}$ mice (Supplemental Figure 12). These findings demonstrate that PBA not only prevents elevation of IOP, but also reduces elevated IOP in older Tg-MYOC ${ }^{Y 437 H}$ mice.

Whole-mount $\gamma$-synuclein staining of the retina demonstrated that PBA rescues RGC loss in Tg-MYOC ${ }^{Y 437 H}$ mice. Untreated $T g$ $M Y O C^{Y 437 H}$ mice lost $21.9 \%$ of RGCs compared with WT litter- 
A

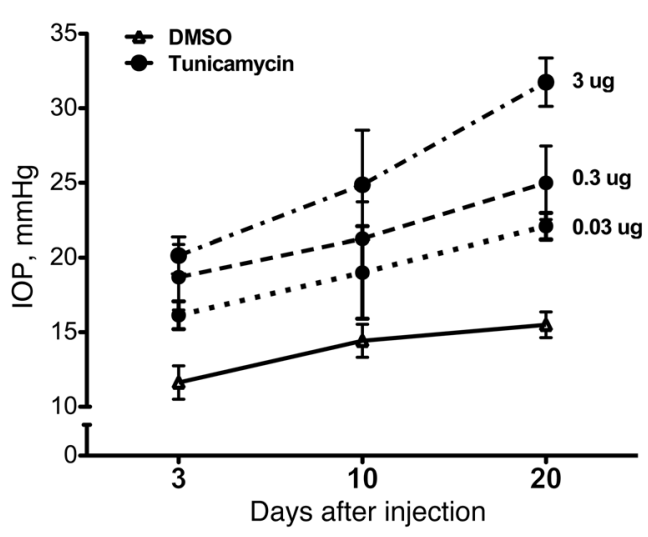

B

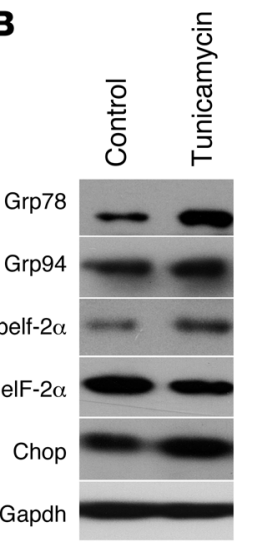

\section{Figure 5}

Chemical induction of ER stress in the TM elevates IOP. (A) Induction of ER stress in the TM of C57BL/6 J by tunicamycin leads to elevated IOP when compared with vehicle control (DMSO). $n=12$ for each group. ${ }^{* \star \star} P<0.005$ versus control. Data are shown as mean \pm SEM. (B) Increased ER stress markers including Grp78, Grp94, Chop, and pelF-2 $\alpha$ were detected in the iridocorneal angle tissues of tunicamycininjected mice by Western blot $(n=4)$. mates, whereas PBA treatment significantly prevented loss of RGCs $(P<0.001$; Figure 6B). Similarly, PBA treatment preserved PERG amplitude in $T g-M Y O C^{Y 437 H}$ mice. Untreated $T g-M Y O C^{Y 437 H}$ mice showed about $33.5 \%$ reduction in PERG amplitude, which was prevented by PBA treatment $(P<0.03$; Figure $6 \mathrm{C})$. We also determined whether PBA prevents neuronal degeneration in Tg-MYOC ${ }^{Y 437 H}$ mice. $T g-M Y O C^{Y 437 H}$ mice lost $20.8 \%$ of their RGC axons compared with WT mice. PBA treatment significantly prevented optic nerve degeneration $(P<0.005$; Figure $6 \mathrm{D})$. These findings indicate that PBA treatment prevents glaucoma phenotypes in $\mathrm{Tg}-M Y O \mathrm{C}^{\mathrm{Y} 437 \mathrm{H}}$ mice.

$P B A$ promotes trafficking and secretion of mutant MYOC. We next sought to determine how PBA protects against glaucoma in $\mathrm{Tg}-\mathrm{MYOC} \mathrm{C}^{\mathrm{Y} 37 \mathrm{H}}$ mice. Since mutations in MYOC inhibit its secretion (17), we investigated whether PBA treatment enhances secretion of MYOC. PBA treatment increased secretion of mutant MYOC by $15 \%$ in cultured TM cells and also in the aqueous humor of Tg-MYOC ${ }^{Y 437 H}$ mice (Figure 7, A and B). Colocalization studies of MYOC with ER marker KDEL revealed that PBA did not alter WT
MYOC localization (Supplemental Figure 13A). However, consistent with increased secretion of mutant myocilin, accumulation of mutant MYOC in the ER was reduced by PBA treatment (Figure 7C). Western blot analysis demonstrated that mutant MYOC forms aggregates in TM cells and that PBA treatment reduces these aggregates (Supplemental Figure 13B). PBA treatment of cultured TM cells expressing mutant MYOC also significantly reduced the loss of TM cells compared with TM cells expressing WT MYOC (Figure 7D). Intracellular MYOC levels and ER stress markers Grp78, Grp94, pelF-2 $\alpha$, active Atf- $6 \alpha$, and Chop were significantly reduced in the iridocorneal angle of PBA-treated $T g-M Y O C^{Y 437 H}$ mice compared with untreated mice (Figure 7E and Supplemental Figure 14). Thus, PBA prevents glaucoma by promoting the secretion of myocilin and depleting intracellular mutant MYOC and reduction of ER stress associated with mutant MYOC.

ER stress is induced in fibroblasts from a Y437HMYOC mutation patient. To determine whether ER stress is induced in POAG patients with the Y437HMYOC mutation, we utilized skin

\section{Figure 6}

Small chemical chaperone rescues glaucoma phenotypes in $\mathrm{Tg}$ MYOC ${ }^{\mathrm{Y} 437 \mathrm{H}}$ mice. (A) Nocturnal IOP measurements, (B) RGC counts, (C) PERG amplitudes, and (D) neuronal degeneration in PBA-treated mice compared with untreated littermates. 2-month-old WT and Tg-MYOC ${ }^{4} 437 \mathrm{H}$ mice were treated with PBA $(20 \mathrm{mM}$, $\sim 10 \mathrm{mg} / \mathrm{mouse} /$ day) in drinking water for 5 weeks and glaucoma phenotypes were compared with those of untreated littermates. $n=8 \mathrm{WT}$ and $16 \mathrm{Tg}-\mathrm{MYOC}^{\mathrm{Y} 437 \mathrm{H}}$ mice. ${ }^{*} P<0.05$ vs. WT; ${ }^{* *} P<0.005$ vs. WT; ${ }^{\dagger} P<0.005$ versus untreated $\mathrm{Tg}-\mathrm{MYOC}^{\mathrm{Y} 437 \mathrm{H}}$. Data are shown as mean \pm SEM.
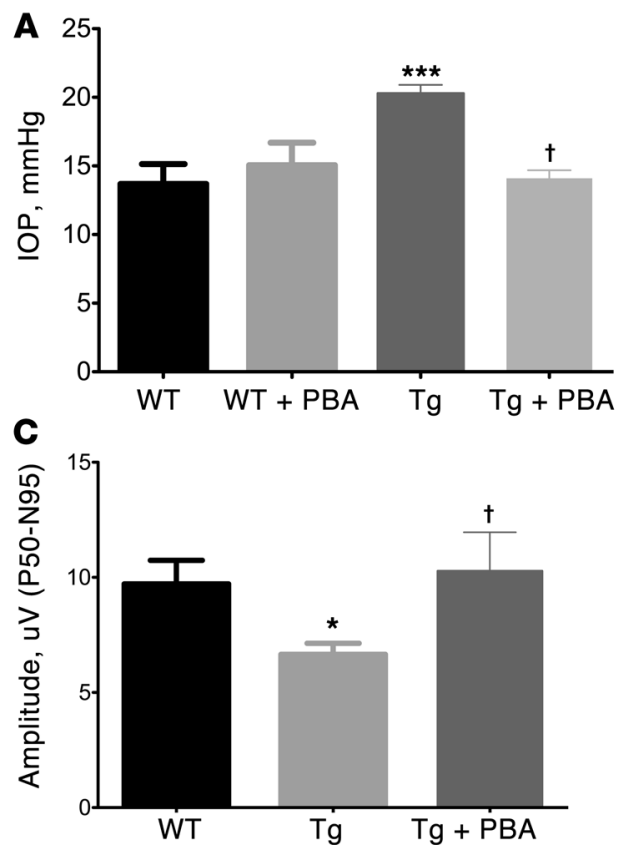

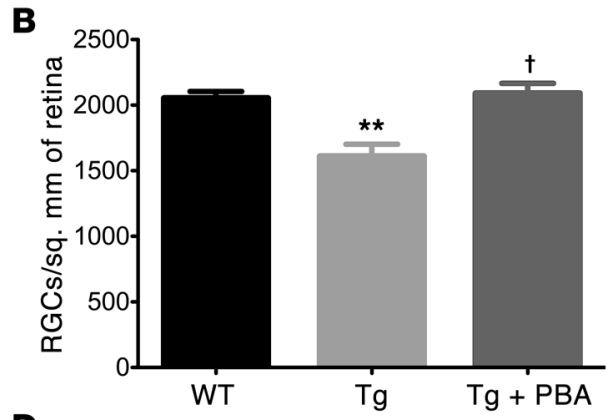

D

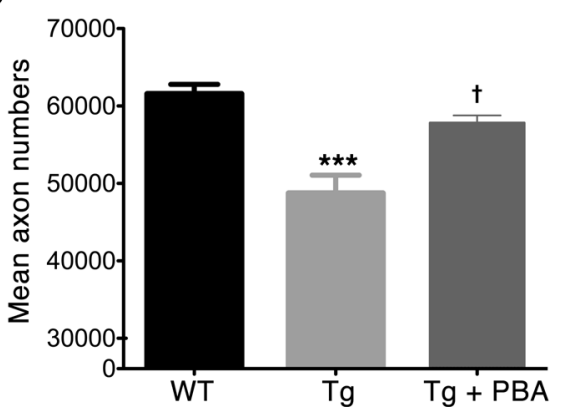



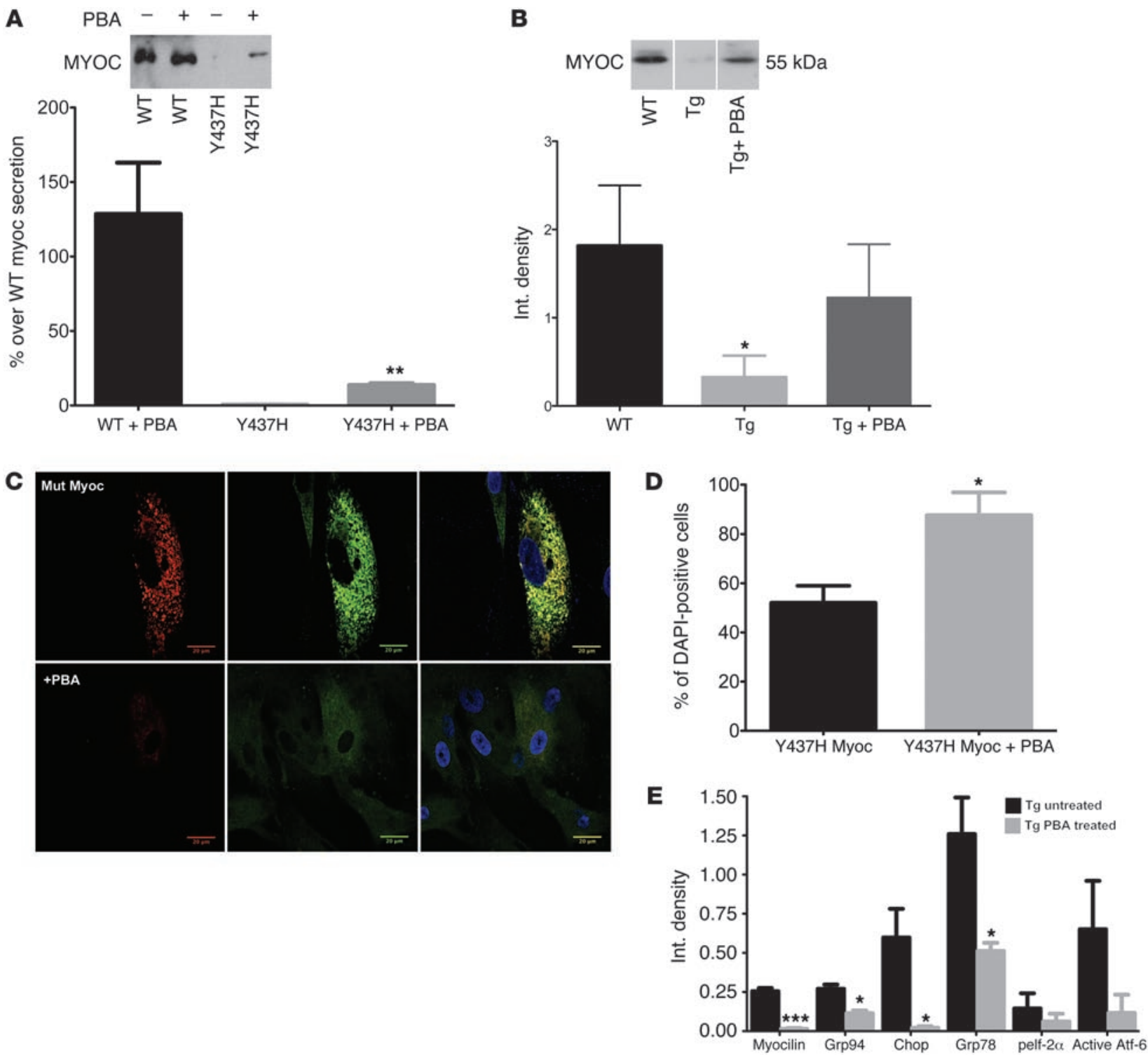

Figure 7

PBA promotes trafficking and secretion of mutant MYOC, thus reducing ER stress in vitro and in vivo. MYOC secretion in the (A) medium or (B) aqueous humor was detected by Western blotting, as shown in the top panel, and analyzed by densitometric analysis, as shown in the bottom panel ( $n=4 \mathrm{WT}, 6$ untreated $T g-M_{Y O C}{ }^{4437 H}$, and 8 PBA treated $T g-M Y O C^{4437 H}$ mice). For myocilin secretion in the aqueous humor, samples were on the same gel but rearranged in the order presented. PBA effect on colocalization of MYOC with ER marker was performed using immunostaining and confocal imaging (C). Scale bars: $20 \mu \mathrm{m}$. PBA treatment rescued loss of TM cells due to mutant MYOC $(n=3)(\mathbf{D})$. Densitometric analysis of ER stress markers in the iridocorneal angle tissue lysates of untreated and treated $T g-M Y O C^{Y 437 H}$ mice $(n=3)(E)$. ${ }^{\star} P<0.05 ;{ }^{* *} P<0.005 ;{ }^{* *} P<0.0005$ versus untreated $T g-M Y O C^{Y 437 H}$. Data are shown as mean \pm SEM.

fibroblasts from a POAG patient with the Y437HMYOC mutation and compared the ER stress markers with age-matched control fibroblasts. Western blot analysis demonstrated that fibroblasts from the Y437HMYOC mutation patient induced ER stress markers, including increased GRP78, GRP94, phosphorylated elf- $2 \alpha$, activated ATF- $\alpha$, and induction of CHOP compared with age-matched control fibroblasts (Figure 8). In addition, fibroblasts obtained from the POAG patient with the MYOC mutation show similar levels of myocilin compared with normal fibroblasts. These data indicate that normal levels of mutant myocilin result in ER stress in humans.

\section{Discussion}

Glaucoma is a complex disease that appears to be influenced by multiple genetic and environmental factors. Mutations in myocilin (MYOC) are an important cause of POAG. Considerable progress has been made in understanding the role of mutant MYOC in glaucoma by using cell culture. However, the in vivo mechanisms leading to MYOC-associated glaucoma are not well understood due to the lack of a faithful animal model. We and others previously attempted to generate a mouse model of glaucoma by knockout of the mouse Myoc gene (8) as well as by knockin of specific human mutations (21). These strategies led to mice that had either no phenotype or a 


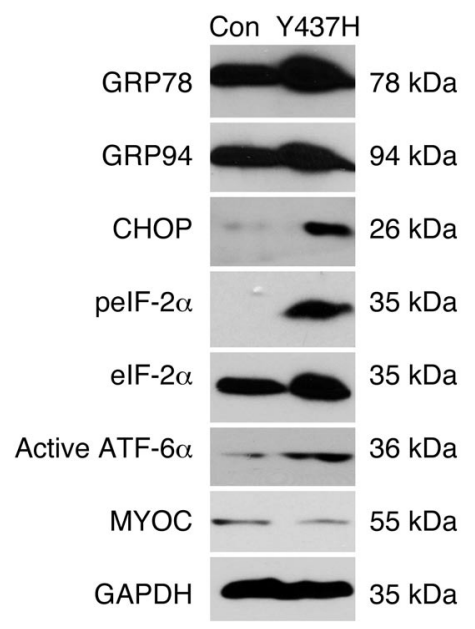

Figure 8

ER stress is induced in fibroblasts from a POAG patient with the Y437HMYOC mutation. Primary fibroblasts from a POAG patient with the Y437HMYOC mutation and age-matched control fibroblasts were examined for ER stress markers by Western blot analysis.

minimal glaucoma phenotype. Here, we report the development of a mouse model that closely replicates the POAG phenotype observed in glaucoma patients. In the current work, we used a CMV promoter to drive the expression of the human MYOC gene containing the $\mathrm{Y} 437 \mathrm{H}$ mutation. We reasoned that the CMV promoter had the potential for relatively high MYOC expression in relevant tissues. The Y437H mutation was selected because human patients with this mutation have early (juvenile) onset of POAG with elevated IOPs (4). A human rather than mouse transgene was inserted due to potential important differences between mouse and human MYOC. The mutant human transgene proved to be expressed in tissues relevant to the development of glaucoma, specifically the iridocorneal angle including the TM and sclera. The transgene is not expressed in the retina of $\mathrm{Tg}-M Y O C^{Y 437 H}$ mice. This pattern is similar to the expression of the endogenous gene. It is known that the $C M V$ promoter does not necessarily lead to ubiquitously expressed transgenes. DNA methylation of the $C M V$ promoter plays an important role in tissue-specific silencing of transgenes (22). Thus, it is possible that lack of transgene expression in retina is due to methylation of the $C M V$ promoter. The study of relevant phenotypes in $T g-M Y O C^{Y 437 H}$ mice reveals development of glaucoma phenotypes closely mimicking those seen in human patients. Notably, adult transgenic mice develop elevated IOP at about 3 months of age and undergo progressive RGC death and optic nerve degeneration. Mutant mice do not display other ocular or nonocular phenotypes. We believe that the $\mathrm{Tg}-M Y O \mathrm{C}^{\mathrm{Y} 437 \mathrm{H}}$ mouse is the first genetic model of MYOC-associated POAG that faithfully replicates the human disorder.

We find it very intriguing that RGC cell death is not linear even though IOP is constantly elevated from 3 months of age. It is possible that initial elevated IOP insult causes cell death of a particular type of RGCs that are the most sensitive to pressure-related damage, while the remaining RGCs are more resistant to cell death by elevated IOP. However, it is important to note that PERG, which is a measurement of RGC function, more dramatically decreases with age (40\% in 4-month-old Tg mice and 60\% in 12-month-old $\mathrm{Tg}-\mathrm{MYOC}^{\mathrm{Y} 437 \mathrm{H}}$ mice). Future studies will be aimed at further understanding loss of RGCs in Tg mice.
The development of Tg-MYOC ${ }^{Y 437 H}$ mice provided the opportunity to investigate the in vivo pathophysiology of MYOC-associated glaucoma. Normal MYOC is secreted, although there are conflicting reports concerning the intracellular localization of MYOC (23-25). In vitro studies indicated that I477N and P370L missense mutations in human MYOC lead to accumulation of the mutant protein in the ER $(11,13)$. Here, we show that $\mathrm{Y} 437 \mathrm{H}$ MYOC accumulates intracellularly in the ER of primary TM cells. In addition, myocilin also accumulates in the ER of TM tissue in vivo, as evident from colocalization of the ER marker KDEL with myocilin in $\mathrm{Tg}-\mathrm{MYOC} \mathrm{C}^{\mathrm{Y} 37 \mathrm{H}}$ mice. Consistent with the nonsecretion and accumulation of mutant MYOC in the ER, MYOC is greatly reduced in the aqueous humor of $\mathrm{Tg}-\mathrm{MYOC} \mathrm{C}^{\mathrm{Y} 37 \mathrm{H}}$ mice compared with controls. The intracellular accumulation of myocilin induces ER stress in the TM of Tg-MYOC ${ }^{Y 437 H}$ mice. To alleviate ER stress, TM cells activate the cytoprotective UPR, as shown by phosphorylation and activation of ER stress sensors as well as downstream signaling pathways, including increased key ER chaperones. Failure to resolve chronic and persistent ER stress may lead to TM loss/dysfunction, which is associated with induction of ER stress-initiated apoptotic transcriptional factor Chop and activation of ER-specific caspase 12 . We observed TM cell loss in 12-month-old Tg-MYOC ${ }^{Y 437 H}$ mice. Interestingly, 3-monthold $\mathrm{Tg}-\mathrm{MYOC}^{\mathrm{Y} 437 \mathrm{H}}$ mice have normal TM cell numbers compared with WT littermates. Furthermore, in primary TM cells, expression of mutant myocilin leads to cell death. The induction of ER stress-initiated cell death signals, including Chop and activated caspase 12, suggests the involvement of ER stress in TM loss. It is interesting to note that older Tg-MYOC ${ }^{Y 437 H}$ mice lose only $30 \%$ of TM and PBA reduces IOP of 12 -month-old $\mathrm{Tg}-\mathrm{MYOC} \mathrm{C}^{\mathrm{Y4} 3 \mathrm{H}}$ mice by $3.5 \mathrm{mmHg}$ compared with $5.5 \mathrm{mmHg}$ in 4-month-old mice. Based on these data, it is possible that elevated IOP in combination with chronic ER stress is involved with TM cell loss observed in older mice. Future studies will be aimed at further understanding the cause of TM loss in $\mathrm{Tg}-M Y O C^{Y 437 H}$ mice. These findings indicate that mutant myocilin induces chronic ER stress, which may lead to dysfunction and/or loss of TM cells in Tg-MYOC ${ }^{Y 437 H}$ mice, thus elevating IOP. Consistent with these findings, chemical induction of ER stress using tunicamycin significantly elevated IOP in normal C57BL/6J mice.

Overexpression of proteins can lead to induction of ER stress. Therefore, it is possible that overexpression of myocilin under the control of the CMV promoter in Tg-MYOC ${ }^{Y 437 H}$ mice may lead to ER stress and elevate IOP. When WT and Y437H human myocilin were overexpressed at comparable levels in the TM of normal mice, only mutant mice developed elevation of IOP and RGC cell death, indicating that only mutant myocilin expression leads to glaucoma. These data are consistent with the previous studies performed by Shepard et al., which demonstrated that transient overexpression of Y437H human MYOC elevates IOP in mice compared with WT human myocilin (10). Furthermore, our data indicate that ER stress is induced prior to IOP elevation based on the following observations. First, mice injected with adenovirus harboring either $\mathrm{Y} 437 \mathrm{H}$ or G364V mutants of human myocilin did not have elevated IOP 24 hours after injections. However, Western blot analysis of iridocorneal angle tissues from these mice demonstrates that $\mathrm{Y} 437 \mathrm{H}$ or G364V mutants of human myocilin induce ER stress (Figure 3E). Second, mice injected with tunicamycin induce ER stress 24 hours after injections but do not elevate IOP (Figure 5B). Third, TM cells expressing only mutant myocilin show ER stress compared with 
TM cells expressing WT myocilin, indicating that mutant myocilin is sufficient to induce ER stress (Supplemental Figure 6). Furthermore, we show that ER stress occurs in the skin fibroblasts of POAG patients with the Y437HMYOC mutation, indicating that a similar phenomenon of ER stress occurs in a human patient with the MYOCY437H mutation. Interestingly, fibroblasts from POAG patients with the Y437HMYOC mutation appear to make low levels of myocilin. but the low levels of myocilin still induce ER stress. Thus, these data indicate that misfolding and not the overexpression of mutant myocilin leads to ER stress.

Mutant myocilin is expressed in tissues throughout the body, yet the only recognized phenotype of mutant myocilin in humans is glaucoma $(3,4)$. Consistent with this phenomenon in humans, $\mathrm{Tg}-\mathrm{MYOC}^{\mathrm{Y} 437 \mathrm{H}}$ mice express human mutant myocilin in heart and kidney at high levels, yet these mice do not show other phenotypes associated with ER stress (data not shown). It is possible that myocilin is misfolded in these tissues; however, these tissues may be able to better handle misfolded myocilin by the UPR pathways. We hypothesize that there are tissue-specific differences in ER stress responses to misfolded myocilin and that malfunction or inefficiency of the ER stress response in the TM leads to cell death. This hypothesis is supported by the findings from a previous study showing significant differences in the ER stress response to mutant myocilin between TM cells and HEK293 cells (11).

We demonstrate that relieving ER stress induced by mutant myocilin in the TM of Tg-MYOC ${ }^{Y 437 H}$ mice prevents glaucoma. We demonstrate that oral administration of PBA not only facilitates secretion of mutant MYOC, but also suppresses ER stress and thus prevents elevated IOP, RGC death, and optic nerve degeneration in $T g-M Y O C^{Y 437 H}$ mice. Previous studies indicate that mutant MYOC is thermally unstable and misfolded. Enhancing proper folding of MYOC by the use of temperature modifications or small chemical chaperones can reverse the pathogenic events in vitro $(11,26-28)$. Our data show that rescue of $\mathrm{Tg}-M Y O \mathrm{C}^{\mathrm{Y} 437 \mathrm{H}}$ mice by PBA is likely due to its chaperone property; this works by enhancing the folding of mutant MYOC leading to increased secretion. Enhanced secretion reduces ER stress and prevents TM cell death. It is remarkable that PBA was able to reduce IOP in 12-month-old mice. Since 12 -monthold mice lose only $30 \%$ of TM cells, it is possible that PBA works on the remaining $70 \%$ of TM cells. However, it is interesting to note that PBA's effects on IOP in 12-month-old mice is less efficient compared with that in 4-month-old mice. PBA treatment decreases IOP by approximately $6 \mathrm{mmHg}$ in 4-month-old $\mathrm{Tg}$ - $M Y O C^{\mathrm{Y} 437 H}$ mice. However, in 12-month-old mice, PBA reduces IOP by approximately $3.8 \mathrm{mmHg}$.

A recent study by Shepard and colleagues demonstrated that expression of glaucomatous mutations in human myocilin caused aberrant peroxisomal localization in TM cells and IOP elevation in mice (10). Human myocilin contains a cryptic carboxyterminal peroxisomal target site (PTS1) that is normally not recognized because WT myocilin is sequestered in the secretory compartment. Mouse myocilin lacks this carboxyterminal PTS1 site. Replacing the human PTS1 site (SKM) with the mouse carboxyl terminal 3 amino acids (LEM) prevented human mutant myocilin from elevating IOP in mice. This concept is further supported by the lack of a glaucoma phenotype in mice expressing mutant mouse myocilin (9). Glaucoma-associated mutations in both human and mouse myocilin cause myocilin misfolding and retention of mutant myocilin in the ER. In addition to our current data, there are also data by others showing that mutant human myocilin activates UPR and
ERAD pathways (11-13). However, although mutant mouse myocilin was not secreted into the aqueous humor and accumulated in the angle of mouse eyes, it did not activate UPR/ERAD and did not elevate IOP in mice (9). Since mutant human MYOC activates the UPR/ERAD pathways and causes mislocalization to peroxisomes, we currently do not know whether one pathway or both pathways are required for damaging the TM leading to IOP elevation. Treatment with the chemical chaperone PBA should promote refolding of mutant myocilin and thereby rescue both pathways. Thus, failure to secrete myocilin, induction of ER stress, and mislocalization of mutant myocilin to peroxisomes appear to be central features of MYOC-associated glaucoma and support the gain-of-function hypothesis of mutant MYOC. This study provides a basis for examining the role of ER stress in POAG patients and evaluating the use of chemical chaperones in the mitigation of POAG.

There are several widely used rodent models of glaucoma, and each has its own strengths and weaknesses $(29,30)$. The naturally occurring DBA/2J mouse model of glaucoma develops pigmentary glaucoma-like changes in the anterior segment, which causes IOP elevation followed by progressive optic nerve damage and RGC loss (31). There also are a number of induced models of ocular hypertension that cause optic nerve and RGC damage, including injection of hypertonic saline into episcleral veins in rats (32), laser photocoagulation of the aqueous outflow pathway in rats and mice (33-35), cautery of extraocular vessels in rats (36) and mice (37), and injection of microbeads to occlude the outflow pathway in rats and mice (38). The current Tg-MYOC ${ }^{Y 437 H}$ model is based on our previous work demonstrating that intraocular injection of mouse eyes with specific adenoviral Myoc expression vectors elevated IOP (10). Our new model uses a mutant human glaucoma gene to mimic many features of POAG including the following: TM damage with an open anterior chamber angle, elevated IOP, progressive optic nerve and RGC damage, and loss of visual function. Unlike in acute models, the pressure-related damage occurs progressively over the course of months. In addition, the damage is synchronous, with similar IOP elevations and damage at a specific age occurring in both eyes (intraindividual) and among littermates (interindividual). We have also shown that therapeutic intervention both early and late in the disease process can protect from glaucomatous damage.

\section{Methods}

Generation of $\mathrm{Tg}-\mathrm{MYOC} \mathrm{C}^{\mathrm{Y} 37 \mathrm{H}}$ transgenic mice. The human MYOC gene was amplified from a human Marathon cDNA Library (Clontech) and cloned into the mammalian expression vector $\mathrm{PCS} 2^{+}$. The $\mathrm{CDNA}$ was modified to introduce a Tyr437His mutation using the QuikChange Mutagenesis Kit (Stratagene). The sequence of the construct was verified with Sanger DNA sequencing. The expression cassette containing the CMV promoter, MYOC cDNA, and SV40 polyA was excised with NotI and SalI restriction enzymes and injected in the pronucleus of freshly fertilized C57BL/6J $\times \mathrm{SJL} / \mathrm{J}$ oocytes (B6SJL; The Jackson Laboratory). The founder was mated with C57BL/6J mice and experiments performed with $\mathrm{F} 2$ and later generations of intercrossed mice B6SJL; $\mathrm{Tg}\left(\mathrm{CMV}-\mathrm{MYOC}^{\mathrm{Y} 437 \mathrm{H}}\right) / \mathrm{Vcs}$ (abbreviated throughout as $T g-M Y O C^{Y 437 H}$ ). Mice were genotyped by $\mathrm{PCR}$ using primers specific to human MYOC. Primers were as follows: hMYOC-Tr-Fr: CGTGCCTAATGGGAGGTCTAT; hMYOC-Tr-Rr: CTGGTCCAAGGTCAATTGGT.

Inbred SJL/J mice carry the $P d e 6 b^{r d 1}$ and Tyr ${ }^{c}$ mutations. To avoid potentially confounding influences of these mutations in $T g-M Y O C^{Y 437 H}$, all mice used in experiments were genotyped for $P d e 6 b^{r d l}$ mutations. Mice that were either WT or heterozygous for $P d e 6 b^{r d 1}$ and pigmented were used in experiments. 
Mouse busbandry. Mice were housed and bred at the University of Iowa Research Animal Facility. Mice were maintained on a 4\% fat NIH 31 diet provided ad libitum and housed in cages containing dry bedding (SoftZorb Enrichment Blend; Northeastern Products). The environment was kept at $21^{\circ} \mathrm{C}$ with a 12 -hour light/12-hour dark cycle. All animal procedures performed in this study complied with the ARVO Statement for the Use of Animals in Ophthalmic and Vision Research and were approved by the University of Iowa Animal Care and Use Committee.

IOP measurements. IOP was measured with a rebound tonometer as described previously (39). Mice were anesthetized using $2.5 \%$ isoflurane plus $100 \%$ oxygen. IOP was measured with a tonometer (TonoLab; Colonial Medical Supply). Daytime IOP was measured between 9 and $11 \mathrm{am}$. The same mice were used to measure nocturnal IOP and IOP were measured in the dark between $11 \mathrm{pm}$ and $1 \mathrm{am}$.

RGCs counting. $\gamma$-Synuclein preferentially stains RGCs, and the total number of surviving RGCs was determined using confocal imaging as described previously $(40,41)$. Whole-mount preparations of retinas were incubated with $0.3 \%$ Triton X-100 overnight and blocked with $5 \%$ goat serum for 1 hour. These retinas were then incubated with $\gamma$-synuclein antibody for 3 hours, followed by standard secondary antibody (goat anti-rabbit 568, 1:200; Invitrogen) incubation. For counting, 5 nonoverlapping images of the midperipheral region were captured from each quadrant at $\times 200$ magnification using a LaserSharp2000 confocal scanning microscope (Bio-Rad). $\gamma$-Synuclein-positive cells were counted in $300 \mu \mathrm{m}^{2}$ area of each image using ImageJ software (NIH).

PERG. PERG was used to objectively measure the function of RGCs by recording amplitudes and latency of N35-P50 and P50-N95 PERG waveforms. Mice were anesthetized with a mixture of $75 \% \mathrm{O}_{2}$ and $25 \% \mathrm{NO}$ and $3.5 \%$ halothane. Mice were then placed on a stainless steel recording table equipped with a hot-water-based warming platform to maintain body temperature, and halothane concentration was decreased to $1.75 \%$. Mice were positioned $20 \mathrm{~cm}$ from the stimulus monitor with their head angle tilted at 45 degrees to provide direct exposure of the grating stimulus to the visual axis of the recorded eye. Pattern ERG responses were evoked using alternating, reversing, black and white vertical stimuli delivered on a CRT monitor with a Roland Consult ERG system (Roland Consult). Each animal was placed at the same fixed position in front of the monitor to prevent recording variability due to animal placement. Stimuli $\left(9^{\circ}\right.$ full field pattern, 1 $\mathrm{Hz}$ frequency, 200 averaged signals with cut-off filter frequencies of 1-30 $\mathrm{Hz}$ ) were delivered in photopic conditions, since slow stimulation speed in mesopic and scotopic conditions can elicit rod-mediated full-field ERG responses, which can completely conceal the PERG responses in rodents. Visually evoked responses were evaluated by measuring amplitudes (N35$\mathrm{P} 50$ and P50-N95) and corresponding implicit times (latency). Implicit times were calculated for N35, P50, and N95 markers in addition to the implicit times for N35-P50 and P50-N95 components.

Assessment of optic nerve damage. Optic nerve neurodegeneration was examined using a paraphenylenediamine (PPD) stain. PPD stains the myelin sheath of all axons and stains the axoplasma of damaged axons differentially (42). Optic nerves were cut and fixed overnight in phosphate-buffered $3 \%$ glutaraldehyde/paraformaldehyde mixture at $4{ }^{\circ} \mathrm{C}$ followed by overnight treatment in $1 \%$ osmium tetroxide at $4{ }^{\circ} \mathrm{C}$. Nerves were then rinsed twice for 10 minutes in $0.1 \mathrm{M}$ phosphate buffer and once in $0.1 \mathrm{M}$ sodium-acetate buffer, then dehydrated in graded ethanol concentrations. After embedding in resin (Eponate-12; Ted Pella), $1-\mu \mathrm{m}$ sections were cut and stained in $1 \%$ PPD for 10 minutes. Optic nerve axons were graded and counted using a previously described method (42). In short, 18 nonoverlapping images covering the entire optic nerve were taken at $\times 1000$ magnification, and axons were counted in the area equal to $10 \%$ of the total nerve cross-sectional area.
Immunostaining. Mouse anterior segment tissues or TM cells were fixed in $4 \%$ formaldehyde and embedded in sucrose. The sections were then blocked with $5 \%$ normal serum. Slides were incubated overnight with primary antibody in $1.5 \%(\mathrm{v} / \mathrm{v})$ normal serum, then washed 3 times with PBS followed by 2-hour incubation in appropriate Alexa Fluor secondary antibodies (1:200; Invitrogen). Sections were subsequently incubated with DAPI for 30 minutes to stain nuclei, washed, and then mounted. Images were captured using a Zeiss 710 confocal imaging system at the University of Iowa Central Microscopy Research Facility. Myocilin antibody used for immunostaining was obtained from Stanislav Tomarev (NIH, Bethesda, Maryland, USA).

Western blot analysis. Anterior segment or iridocorneal angle tissues were carefully dissected out and lysed in lysis buffer. Cellular proteins were separated on denaturing polyacrylamide gels and then transferred to PVDF membranes by electrophoresis. Blots were blocked with $5 \%$ non-fat milk for 1 hour. The blots were then incubated overnight with specific primary antibodies. The membranes were washed with Tris-buffered saline/Tween buffer (TBST) and incubated with a corresponding horseradish peroxidase-conjugated secondary antibody. The proteins were then visualized in an $\mathrm{x}$-ray developer using ECL detection reagents (SuperSignal West Femto Maximum Sensitivity Substrate; Pierce Biotechnology). To ensure equal protein loading, the same blot was subsequently incubated with a GAPDH monoclonal antibody (Cell Signaling Technology Inc.). Quantitation was done using ImageJ software (NIH). To examine MYOC secretion, $50 \mu \mathrm{l}$ of conditioned medium was subjected to Western blot analysis. To examine myocilin in the aqueous humor, approximately $2 \mu \mathrm{l}$ of aqueous humor was isolated from each untreated Tg-MYOC ${ }^{Y 437 H}(n=6)$, PBA-treated Tg-MYOC ${ }^{Y 437 H}(n=6)$, and WT mouse $(n=5)$. Antibodies for GRP78, GRP94, pelF- $2 \alpha$, elF-2 $\alpha$, Ire1, and $\mathrm{CHOP}$ were purchased from Cell Signaling Technology Inc. Antibodies for $\gamma$-synuclein, caspase 12, and XBP-1 were from Abcam Inc., and antibodies for ATF-6 were bought from Imgenex Corp. MYOC antibody (sc-137233) was bought from Santa Cruz Biotechnology Inc. For analysis of intracellular MYOC aggregates, cells were lysed in buffer containing 0.5\% Triton X-100, and soluble fractions were analyzed by Western blotting.

Culture of buman TM cells and human fibroblasts. Human TM cells were obtained from Alcon Research Ltd. and grown as described previously (43). Cell cultures were maintained in DMEM supplemented with $10 \%$ FBS (Hyclone Laboratories), $2 \mathrm{mM}$ L-glutamine, penicillin (10,000 units/ml), and streptomycin $(10 \mu \mathrm{g} / \mathrm{ml})$ (Gibco BRL; Invitrogen). Skin biopsy was obtained from $Y 437 \mathrm{H}$ heterozygous MYOC mutation POAG patient and age-matched control human donor. Human fibroblasts were grown in DMEM supplemented with $10 \%$ FBS. Adenoviral vectors were obtained from the Gene Transfer Vector Core, University of Iowa $(9,17)$. For adenoviral transfections, equal numbers of TM cells were grown in 12-well plates for 8-10 days. Cells were infected with adenovirus at $20 \mathrm{PFU} /$ cell for 1 hour in serum-free DMEM. 4-PBA ( $5 \mathrm{mM}$ ) was added to cells infected with adenovirus expressing WT MYOC or Y437H MYOC, and TM cells were grown for 5 days. The culture medium containing 4-PBA was changed daily. The cell lysates and medium were collected and subjected to further analysis.

Anterior chamber injections. For anterior chamber injections, the animals were anesthetized with a mouse anesthesia cocktail (ketamine $[73 \mathrm{mg} / \mathrm{kg}]$, and xylazine $[1.8 \mathrm{mg} / \mathrm{kg}])$. Adenoviral vectors $\left(3 \times 10^{9} \mathrm{PFU} /\right.$ eye $)$ or tunicamycin $(0.03,0.3$, and $3 \mu \mathrm{g} /$ eye) were injected in the anterior chamber in a volume of $2 \mu \mathrm{l}$ into both eyes of each animal. Mice that developed eye abnormalities were excluded from further study. DMSO injections were used as vehicle control for tunicamycin.

PBA treatments. 2-month-old WT $(n=8)$ and Tg-MYOC ${ }^{Y 437 H}(n=13)$ littermates were divided into 2 groups; 1 group was treated with $20 \mathrm{mM}$ PBA in the drinking water, whereas the other group was given water without the drug. Pharmaceutical-grade 4-PBA sodium salt was purchased from Scandinavian Formulas. 4-PBA sodium salt is soluble in water and thus was 
added freshly to the drinking water every week for 5 weeks. Water intake measurements were made, and both treated and untreated mice consumed equal amounts of water. Mice drank an average of $3.2 \mathrm{ml}$ of water containing the drug every day. Therefore, each mouse received an average of $10 \mathrm{mg} / \mathrm{mouse} / \mathrm{d}$. Since $\mathrm{Tg}-\mathrm{MYOC} \mathrm{C}^{\mathrm{Y} 437 \mathrm{H}}$ mice develop elevated IOP between 2 and 3 months of age, we started PBA treatment at 2 months and examined IOP after 5 weeks of treatment. For treatment of older $T g-M Y O C^{Y 437 H}$ mice, PBA dosing was done as described above, except the treatment was started at 12 months of age. After 5 weeks of treatment, mice were sacrificed and glaucoma phenotypes were compared between these groups by methods described above.

$R T$-PCR. Total RNA was extracted from iridocorneal angle tissues using a commercial kit (RNeasy Mini Kit; QIAGEN), and cDNA was synthesized using reverse transcriptase and random hexamer primers. PCR was performed with the following primers: GAPDH primers (forward, 5'-GTGTCCGTCGTGGATCTGA-3'; reverse: 5'-CCTGCTTCACCACCTTCTTg-3'); mouse Myoc primers (forward, 5'-GCCATCCAAGACCTTCAGAG-3'; reverse: 5'-AGATCCCTGGTTTGGGTCTC-3'); human Y437H MYOC primers (forward, 5' GAGTAAGGCAAGAAAATGAGAATC-3'; reverse: 5'-CCTCTCCACTCCTGAGATAGC-3')

TEM. Mice were perfusion fixed with $4 \%$ formaldehyde, and the eyes were enucleated. The anterior chamber was carefully dissected and fixed in $1.5 \%$ paraformaldehyde and $1.5 \%$ glutaraldehyde, in $100 \mathrm{mM}$ sodium cacodylate buffer ( $\mathrm{pH}$ 7.2), for at least 24 hours. Eyes were then processed for TEM. Briefly, after the aldehyde fixation, a $1 \%$ osmium tetroxide in $100 \mathrm{mM}$ sodium cacodylate buffer postfixation was performed followed by ethanol dehydration and embedding of the samples in Eponate 812 resin. Sections $(90 \mathrm{~nm})$ were cut using an ultramicrotome (EM UC6; Leica) and stained with uranyl acetate and Reynolds lead citrate. Images were recorded with a transmission electron microscope (JEM-1230; JEOL) equipped with a $2 \mathrm{~K} \times 2 \mathrm{~K}$ CCD camera (USC1000; Gatan Inc.) at the University of Iowa Central Microscopy Research Facility. Iridocorneal angle images were taken at $\times 80$ magnification, which covered the entire area of TM and the Schlemm canal in 1 image. The total number of TM cells was counted in 1 field of $5 \mathrm{WT}$ and $6 \mathrm{Tg}-\mathrm{MYOC}^{\mathrm{Y} 437 \mathrm{H}}$ mice. To examine the variability between different regions of TM, serial sections of iridocorneal angle in $2 \mathrm{WT}$ and 2 $\mathrm{Tg}-\mathrm{MYOC} \mathrm{C}^{\mathrm{Y} 37 \mathrm{H}}$ were performed and TM cells were counted in these sections. TM cell count was similar in these serial sections; thus for the rest of the study, 1 section/eye was used to count TM cells.
Cornea thickness measurements. An ultrasound pachymeter (Corneo-Gage Plus; Sonogage) was used to measure murine CCT as described previously (44).

Mouse slit-lamp examination and gonioscopy. Anterior chamber phenotypes were assayed with a slit-lamp (SL-D7; Topcon) and photodocumented with a digital camera (D100; Nikon) as described previously (45). Gonioscopy was performed with a 2-mm gonioprism (Ocular Instruments). All ocular examinations were conducted on conscious mice except for gonioscopy, which used mice anesthetized with a mixture of ketamine $(100 \mathrm{mg} / \mathrm{kg})$ and xylazine $(10 \mathrm{mg} / \mathrm{kg})$.

Statistics. For comparisons between 2 groups, the unpaired Student's $t$ test was used. For comparisons among 3 or more groups, 1-way ANOVA followed by Bonferroni's test was applied. For comparison of IOP, RGC, and optic nerve axonal degeneration between different age groups, 2-way ANOVA was used. $P<0.05$ was considered significant.

\section{Acknowledgments}

This work was supported by funding from the following grants and organizations: NIH (R01 EY10564 to V.C. Sheffield and E.M. Stone); the Carver Endowment for Molecular Ophthalmology; Research to Prevent Blindness (New York, New York, USA); and VA Center for Prevention and Treatment of Vision Loss (Iowa City, Iowa, USA). V.C. Sheffield and E.M. Stone are investigators of the Howard Hughes Medical Institute. The authors thank Allan Shepard for supplying human TM cells. The authors also thank the University of Iowa's Central Microscopy Research Facilities for helping with immunostaining and TEM studies. We thank Arti Sharma for critical review of the manuscript. The authors thank Geoff Lively for technical help with CCT measurements. The authors would like to thank Stanislav Tomarev (NIH) for providing myocilin antibody. The authors are very thankful to John $\mathrm{H}$. Fingert for providing fibroblasts from human POAG patients.

Received for publication March 25, 2011, and accepted in revised form June 1, 2011.

Address correspondence to: Val C. Sheffield, Department of Pediatrics, College Of Medicine 440 EMRB, University of Iowa, Iowa City, Iowa 52242, USA. Phone: 319.335.6898; Fax: 319.335.7588; E-mail: val-sheffield@uiowa.edu.
1. Quigley HA. Number of people with glaucoma worldwide. BrJ Ophthalmol. 1996;80(5):389-393.

2. Stone EM, et al. Identification of a gene that causes primary open angle glaucoma. Science. 1997;275(5300):668-670.

3. Swiderski RE, et al. Localization of MYOC transcripts in human eye and optic nerve by in situ hybridization. Invest Ophthalmol Vis Sci. 2000;41(11):3420-3428.

4. Kwon YH, Fingert JH, Kuehn MH, Alward WL. Primary open-angle glaucoma. $N$ Engl J Med. 2009;360(11):1113-1124.

5. Wiggs JL, Vollrath D. Molecular and clinical evaluation of a patient hemizygous for TIGR/MYOC. Arch Ophthalmol. 2001;119(11):1674-1678.

6. Morissette J, et al. Homozygotes carrying an autosomal dominant TIGR mutation do not manifest glaucoma. Nat Genet. 1998;19(4):319-321.

7. Gould DB, et al. Genetically increasing Myoc expression supports a necessary pathologic role of abnormal proteins in glaucoma. Mol Cell Biol. 2004;24(20):9019-9025.

8. Kim BS, et al. Targeted Disruption of the Myocilin Gene (Myoc) Suggests that Human GlaucomaCausing Mutations Are Gain of Function. Mol Cell Biol. 2001;21(22):7707-7713.
9. Gould DB, Reedy M, Wilson LA, Smith RS, Johnson RL, John SW. Mutant myocilin nonsecretion in vivo is not sufficient to cause glaucoma. Mol Cell Biol. 2006;26(22):8427-8436.

10. Shepard AR, et al. Glaucoma-causing myocilin mutants require the Peroxisomal targeting signal1 receptor (PTS1R) to elevate intraocular pressure. Hum Mol Genet. 2007;16(6):609-617.

11. Liu Y, Vollrath D. Reversal of mutant myocilin nonsecretion and cell killing: implications for glaucoma. Hum Mol Genet. 2004;13(11):1193-1204.

12. Yam GH, Gaplovska-Kysela K, Zuber C, Roth J. Aggregated myocilin induces russell bodies and causes apoptosis: implications for the pathogenesis of myocilin-caused primary open-angle glaucoma. Am J Pathol. 2007;170(1):100-109.

13. Joe MK, Sohn S, Hur W, Moon Y, Choi YR, Kee C. Accumulation of mutant myocilins in ER leads to ER stress and potential cytotoxicity in human trabecular meshwork cells. Biochem Biophys Res Commun. 2003;312(3):592-600.

14. Yoshida H. ER stress and diseases. FEBS J. 2007; 274(3):630-658.

15. Sacca SC, Rolando M, Marletta A, Macri A, Cerqueti P, Ciurlo G. Fluctuations of intraocular pressure during the day in open-angle glaucoma, normal- tension glaucoma and normal subjects. Ophthalmologica. 1998;212(2):115-119.

16. Wolfs RC, Klaver CC, Vingerling JR, Grobbee DE, Hofman A, de Jong PT. Distribution of central corneal thickness and its association with intraocular pressure: The Rotterdam Study. Am J Ophthalmol. 1997;123(6):767-772.

17. Jacobson N, et al. Non-secretion of mutant proteins of the glaucoma gene myocilin in cultured trabecular meshwork cells and in aqueous humor. Hum Mol Genet. 2001;10(2):117-125.

18. Schroder M, Kaufman RJ. The mammalian unfolded protein response. Annu Rev Biochem. 2005;74:739-789.

19. Caballero M, Rowlette LL, Borras T. Altered secretion of a TIGR/MYOC mutant lacking the olfactomedin domain. Biochim Biophys Acta. 2000;1502(3):447-460.

20. Ozcan U, et al. Chemical chaperones reduce ER stress and restore glucose homeostasis in a mouse model of type 2 diabetes. Science. 2006; 313(5790):1137-1140

21. Zhou Y, Grinchuk O, Tomarev SI. Transgenic mice expressing the Tyr437His mutant of human myocilin protein develop glaucoma. Invest Ophthalmol Vis Sci. 2008;49(5):1932-1939.

22. Brooks AR, Harkins RN, Wang P, Qian HS, Liu P, 
Rubanyi GM. Transcriptional silencing is associated with extensive methylation of the CMV promoter following adenoviral gene delivery to muscle. J Gene Med. 2004;6(4):395-404.

23. Ueda J, Wentz-Hunter KK, Cheng EL, Fukuchi T, Abe H, Yue BY. Ultrastructural localization of myocilin in human trabecular meshwork cells and tissues. J Histochem Cytochem. 2000;48(10):1321-1330.

24. O'Brien ET, Ren X, Wang Y. Localization of myocilin to the golgi apparatus in Schlemm's canal cells. Invest Ophthalmol Vis Sci. 2000;41(12):3842-3849.

25. Sohn S, et al. Expression of wild-type and truncated myocilins in trabecular meshwork cells: their subcellular localizations and cytotoxicities. Invest $O p h$ thalmol Vis Sci. 2002;43(12):3680-3685.

26. Yam GH, Gaplovska-Kysela K, Zuber C, Roth J. Sodium 4-phenylbutyrate acts as a chemical chaperone on misfolded myocilin to rescue cells from endoplasmic reticulum stress and apoptosis. Invest Ophthalmol Vis Sci. 2007;48(4):1683-1690.

27. Burns JN, Orwig SD, Harris JL, Watkins JD, Vollrath D, Lieberman RL. Rescue of glaucoma-causing mutant myocilin thermal stability by chemical chaperones. ACS Chem Biol. 2010;5(5):477-487.

28. Jia LY, et al. Correction of the disease phenotype of myocilin-causing glaucoma by a natural osmolyte. Invest Ophthalmol Vis Sci. 2009;50(8):3743-3749.

29. McKinnon SJ, Schlamp CL, Nickells RW. Mouse models of retinal ganglion cell death and glaucoma. Exp Eye Res. 2009;88(4):816-824.

30. Pang IH, Clark AF. Rodent models for glaucoma retinopathy and optic neuropathy. J Glaucoma. 2007;16(5):483-505.

31. Libby RT, et al. Inherited glaucoma in DBA/2J mice: pertinent disease features for studying the neurodegeneration. Vis Neurosci. 2005;22(5):637-648.

32. Morrison JC, Moore CG, Deppmeier LM, Gold BG, Meshul CK, Johnson EC. A rat model of chronic pressure-induced optic nerve damage. Exp Eye Res. 1997;64(1):85-96.

33. Levkovitch-Verbin H, Quigley HA, Martin KR, Valenta D, Baumrind LA, Pease ME. Translimbal laser photocoagulation to the trabecular meshwork as a model of glaucoma in rats. Invest Ophthalmol Vis Sci. 2002;43(2):402-410.

34. Aihara M, Lindsey JD, Weinreb RN. Experimental mouse ocular hypertension: establishment of the model. Invest Ophthalmol Vis Sci. 2003; 44(10):4314-4320.

35. Grozdanic SD, Betts DM, Sakaguchi DS, Allbaugh RA, Kwon YH, Kardon RH. Laser-induced mouse model of chronic ocular hypertension. Invest Ophthalmol Vis Sci. 2003;44(10):4337-4346.

36. Garcia-Valenzuela E, Shareef S, Walsh J, Sharma SC. Programmed cell death of retinal ganglion cells during experimental glaucoma. Exp Eye Res. 1995;61(1):33-44.

37. Ruiz-Ederra J, Verkman AS. Mouse model of sustained elevation in intraocular pressure produced by episcleral vein occlusion. Exp Eye Res. 2006;82(5):879-884.

38. Sappington RM, Carlson BJ, Crish SD, Calkins
DJ. The microbead occlusion model: a paradigm for induced ocular hypertension in rats and mice. Invest Ophthalmol Vis Sci. 2010;51(1):207-216.

39. Kim CY, Kuehn MH, Anderson MG, Kwon YH. Intraocular pressure measurement in mice: a comparison between Goldmann and rebound tonometry. Eye (Lond). 2007;21(9):1202-1209.

40. Kuehn MH, Kim CY, Jiang B, Dumitrescu AV, Kwon YH. Disruption of the complement cascade delays retinal ganglion cell death following retinal ischemia-reperfusion. Exp Eye Res. 2008;87(2):89-95.

41. Soto I, et al. Retinal ganglion cells downregulate gene expression and lose their Axons within the optic nerve head in a mouse glaucoma model. J NeuroSci. 2008;28(2):548-561.

42. Anderson MG, Libby RT, Gould DB, Smith RS, John SW. High-dose radiation with bone marrow transfer prevents neurodegeneration in an inherited glaucoma. Proc Natl Acad Sci U S A. 2005;102(12):4566-4571.

43. Wordinger RJ, et al. Effects of TGF-beta2, BMP-4, and gremlin in the trabecular meshwork: implications for glaucoma. Invest Ophthalmol Vis Sci. 2007;48(3):1191-1200.

44. Lively GD, Koehn D, Hedberg-Buenz A, Wang K, Anderson MG. Quantitative trait loci associated with murine central corneal thickness. Physiol Genomics. 2010;42(2):281-286.

45. Trantow CM, et al. Lyst mutation in mice recapitulates iris defects of human exfoliation syndrome. Invest Ophthalmol Vis Sci. 2009;50(3):1205-1214. 